The Psychological Record, 2008, 58, 269-286

\title{
DERIVED AVOIDANCE LEARNING: TRANSFORMATION OF AVOIDANCE RESPONSE FUNCTIONS IN ACCORDANCE WITH SAME AND OPPOSITE RELATIONAL FRAMES
}

\author{
Simon Dymond \\ Swansea University \\ Bryan Roche \\ National University of Ireland, Maynooth \\ John P. Forsyth \\ University at Albany, State University of New York \\ Robert Whelan \\ University College Dublin \\ Julia Rhoden \\ Swansea University
}

\begin{abstract}
Two experiments were designed to replicate and extend previous findings on the transformation of avoidance response functions in accordance with the relational frames of Same and Opposite. Participants were first exposed to nonarbitrary and arbitrary relational training and testing. Next, during avoidance conditioning, one stimulus from the relational network signaled a simple avoidance response that cancelled a scheduled presentation of an aversive image and sound. The majority of participants who met the criteria for conditioned avoidance also demonstrated derived avoidance. Experiment 1 showed that detailed instructions were not necessary for derived transformation to occur. Experiment 2 showed that more complex patterns of transformation may emerge when another stimulus from the relational network signaled the avoidance response. Implications for understanding clinically significant avoidance behavior are discussed.
\end{abstract}

\footnotetext{
Some of the material from this article was submitted as part of Julia Rhoden's master's degree at the Swansea University, conducted under the supervision of Simon Dymond. We thank Jordan Randell for assistance with data collection and Chris Ninness and two anonymous reviewers for helpful comments.

Address correspondence to Simon Dymond, Department of Psychology, Swansea University, Singleton Park, Swansea SA2 8PP, United Kingdom. E-mail: s.o.dymond@swansea.ac.uk
} 
Explaining the acquisition of avoidance behavior that lacks a history of direct conditioning with regard to the aversive object or event represents a challenge to all conditioning accounts of psychopathology (see Rachman, 1977, 1991). Contemporary associative learning accounts, for instance, acknowledge the role played by language and verbal processes in explaining the etiology of indirectly acquired avoidance behavior. Field (2006) outlined mechanisms other than direct Pavlovian conditioning that may lead to the acquisition of a conditioned response. These so-called pathways to fear (p. 867) include vicarious (i.e., observational) learning and "verbal information," such as instructions. Similarly, Lovibond (2006) emphasized the role of verbal expectancies or "propositional knowledge" (p. 126) in mediating human avoidance learning. To a large extent, associative accounts such as these represent a significant departure from traditional Pavlovian models of psychopathology by making the controversial yet tantalizing suggestion that conditioning with humans may, in some as yet undefined way, differ from that seen with nonhumans (for reviews, see Dawson and Schell, 1987; DeHouwer, Vandorpe, \& Beckers, 2004; Lovibond \& Shanks, 2002). For present purposes, however, it is noteworthy that such accounts do not offer a functional definition of what is meant by "verbal" or "language." Indeed, according to some strict associative accounts verbal information is assumed to have its effects through associative learning (Field, 2006, p. 868).

Contemporary approaches to the behavior analysis of language and cognition define a "verbal" stimulus as one that acquires its effects by virtue of its participation in a relational frame (see Dymond \& Barnes, 1997; Wilson \& Blackledge, 2000). By functionally defining verbal events in this way, it is possible to understand indirectly acquired human avoidance learning in terms of two key concepts: derived relational responding and the transformation of stimulus functions (Dymond \& Rehfeldt, 2000; Forsyth, Eifert, \& Barrios, 2006).

Derived relational responding is the phenomenon whereby, after a series of interrelated discriminations is learned, the stimuli often become related to each other in ways not explicitly trained. To illustrate, if choosing Stimulus B in the presence of Stimulus A is taught (i.e., A-B), and choosing Stimulus $\mathrm{C}$ in the presence of Stimulus B (i.e., B-C) is also taught, it is highly likely that relations will emerge between $\mathrm{B}$ and $\mathrm{A}, \mathrm{C}$ and $\mathrm{B}, \mathrm{A}$ and $\mathrm{C}$, and $\mathrm{C}$ and $\mathrm{A}$ in the absence of any further training. Perhaps one of the most interesting aspects of research on derived relational responding is the transformation of functions phenomena, which involves establishing a particular behavioral response or function for one member of an equivalence relation and then observing that the function emerges for one or more additional members of the same relation, without further training (Dymond \& Rehfeldt, 2000).

In one well-cited demonstration of a transformation of avoidance functions, Augustson and Dougher (1997) first trained and tested participants for the emergence of two four-member equivalence relations (i.e., A1-B1-C1-D1 and A2-B2-C2-D2). Using a baseline Pavlovian conditioning procedure, one member of one class was paired with shock (B1) while one member of the other class was presented without shock (B2). A differential, signaled avoidance task was then introduced wherein shock was avoided if a participant made a key pressing response to the stimulus previously associated with shock. The remaining stimuli from both classes were then presented but in the absence of shock. Consistent with predictions, stimulus presentations from 
the "aversive class" (i.e., A1-B1-C1-D1) evoked avoidance responses, whereas stimulus presentations from the "nonaversive class" (i.e., A2-B2-C2-D2) did not. All participants showed evidence of this differential transformation of avoidance-evoking functions to all members of the aversive class but not to the nonaversive class. This study was the first to show the emergence of avoidance responding to stimuli that had no direct relational history with aversive events and thus helps to explain how avoidance behaviors may develop in the absence of direct aversive conditioning.

Equivalence relations represent just one of several forms of derived relational responding that might occur between stimuli and events. Thus, there are likely many more ways for derived avoidance responses to emerge in the world outside the laboratory than those highlighted by Augustson and Dougher (1997). Several studies conducted under the rubric of relational frame theory have since provided evidence that it is possible for human participants to respond in accordance with relations other than equivalence, such as same and opposite (e.g., Dymond \& Barnes, 1996; Roche \& Barnes, 1997; Steele \& Hayes, 1991; Whelan \& Barnes-Holmes, 2004).

In a recent study, the current authors demonstrated that human avoidance responses may participate in complex relational networks of same and opposite (Dymond, Roche, Forsyth, Whelan, \& Rhoden, 2007). Specifically, participants were first exposed to nonarbitrary and arbitrary relational training and testing in order to establish Same and Opposite relations among arbitrary, nonword stimuli. The arbitrary relational training tasks were Same-A1-B1, Same-A1-C1, Opposite-A1-B2, and Opposite-A1-C2, which yielded the following eight testing tasks: Same-B1-C1, Same-C1-B1, Same-B2-C2, SameC2-B2, Opposite-B1-C2, Opposite-C2-B1, Opposite-B2-C1, and Opposite-B1-C2. Next, an avoidance conditioning procedure was used to establish one member of the relational network (B1) as a discriminative stimulus ( $\mathrm{S}^{\mathrm{D}}$ ) for avoidance responding (i.e., pressing the computer space bar to avoid upcoming aversive images and sounds) and another (B2) as discriminative for nonavoidance responding (i.e., not pressing the space bar and not viewing nonaversive images). After acquisition of the avoidance and nonavoidance responses, participants were tested for a transformation of functions with presentations of $\mathrm{C} 1$ and $\mathrm{C} 2$. All participants who met the criteria for conditioned avoidance also showed derived avoidance, in that they emitted avoidance responses in the presence of $\mathrm{C} 1$ but not in the presence of C2. The findings of Dymond et al. extended the previous findings of Augustson and Dougher (1997) to the relational frames of same and opposite and by employing aversive images and sounds as the aversive events.

The present study sought to replicate and extend our earlier findings in the following two ways. First, a possible limitation of the Dymond et al. (2007) procedure was that relatively detailed instructions were used to initiate contact with the negative reinforcement schedule used in the avoidance-conditioning phase. Specifically, participants were instructed that they should "learn to cancel certain pictures and sounds before they are presented, by pressing the space-bar." It is possible, therefore, that the instruction to emit the avoidance response in the presence of one stimulus but not the other may have facilitated contact with the avoidance contingency and subsequently influenced derived transformation. That is, because of the instruction to cancel certain upcoming pictures and sounds, participants may, at the outset of avoidance conditioning, simply have viewed all stimulus presentations (i.e., not emitted the avoidance 
response) and hence responding may not have come under the direct control of the avoidance schedule. Previous research on human avoidance has not seen it necessary to provide such explicit instructions in order to acquire stable rates of avoidance (Lejuez, O'Donnell, Wirth, Zvolensky, \& Eifert, 1998), and similarly, previous research on transformation of function has shown that detailed instructions are not necessary for derived transformation to occur (e.g., Dymond \& Barnes, 1998). Therefore, Experiment 1 was designed to examine whether the derived transformation effect seen in Dymond et al. would be replicated with modified instructions used in the avoidanceconditioning phase. Second, in Experiment 2, the discriminative stimuli for avoidance and nonavoidance responding, respectively, were varied to examine more complex patterns of transformation (e.g., Roche \& Barnes, 1997).

\section{Experiment 1}

\section{Method}

\section{Participants}

Thirteen participants, aged 22 to 44 years old, were recruited via bulletin board announcements or personal contacts from the undergraduate and postgraduate community at the University of Wales, Swansea, and received $£ 5$ (approximately \$10) after completion of the study. All procedures underwent ethics review and were approved prior to experimentation.

\section{Materials}

A computer program written in Visual Basic 6.0 was used to control all stimulus presentations and to record all responses. Visual and auditory stimuli were selected from the International Affective Picture System (IAPS; Lang, Bradley, \& Cuthbert, 2005) and the International Affective Digitized Sounds (IADS; Bradley \& Lang, 1999) for use as aversive and nonaversive stimuli during the avoidance conditioning and transformation testing phases. On the basis of our previous research, a total of 20 photographs, 10 aversive (e.g., bodily mutilations) and 10 nonaversive (e.g., landscapes), and 10 aversive sounds (e.g., a woman screaming) were selected. ${ }^{1}$

Two stimuli were used as contextual cues for Same (i.e., ) ()), and Opposite (i.e., Đo), respectively. Eight nonsense syllables were employed as sample and comparisons during relational training and testing (i.e., CUG, JOM, ZID, PAF, MEL, LEB, VEP, FIH). These are labeled, in the interests of clarity, with alphanumerics; A1, B1, C1, B2, C2, N1, N2, and N3. The N1, N2, and N3 stimuli were employed as foils; their selection was never reinforced.

\section{Procedure}

General procedure. On arrival at the laboratory, participants signed a consent form, acknowledging the distasteful nature of some of the stimuli to be used

IAPS and IADS identifiers: (Pictures) 1333, 1731, 1811, 1812, 1999, 2791, 2840, 3000, 3010, 3030, 3051, 3060, 3061, 3062, 3063, 3064, 3068, 5260, 5480, 5300; (Sounds) 276, 277, 278, 279, 285, 286, 290, 292, 380, 423. 
during the avoidance conditioning phase and indicating that they did not have a history of psychopathology. Next, participants were seated comfortably at a table in front of a computer in a small experimental room. The experiment began with the following instructions displayed on the computer screen:

Thank you for agreeing to participate in this study. You will be presented with a series of images or nonsense words on the top half of the screen from left to right. Then you will be presented with 5 images or nonsense words on the bottom of the screen. Your task is to observe the images or words that appear from left to right and drag one of these images or words from the bottom to the blank, yellow square. Click and hold the mouse over the image or word to drag it to the blank square. To confirm your choice, click "Finish Trial." If you wish to make another choice, then click "Start Again." Sometimes you will receive feedback on your choices, but at other times you will not. Your aim is to get as many tasks correct as possible. It is always possible to get a task correct, even if you are not given feedback.

Clicking on a check box at the bottom of the screen cleared the instruction screen, and after a 3-s interval, Phase 1 commenced.

During all nonarbitrary and arbitrary relational training and testing phases the computer screen was divided into two areas: the top half was blue, the remainder gray. The sample appeared on the left upper portion of the screen, after $1 \mathrm{~s}$ the contextual cue appeared in the upper center, and after a further $1 \mathrm{~s}$ a "blank" comparison square appeared $1 \mathrm{~s}$ later on the right upper portion of the screen. Five comparison stimuli appeared simultaneously on the lower section of the screen. The location of the comparison stimuli across the bottom of the screen was randomized across trials.

The first two phases consisted of nonarbitrary relational training and testing designed to establish contextual cues for generalized Same and Opposite responding. The third phase involved arbitrary relational training during which participants were trained to relate a series of arbitrary stimuli (i.e., nonsense syllables) in the presence of the Same and Opposite cues to establish a contextually controlled relational network of arbitrary stimuli. The fourth phase involved arbitrary relational testing and was used to probe for the emergence of derived relations among the arbitrary stimuli in the network. Across all phases, the relational completion procedure (Dymond et al., 2007; Dymond \& Whelan, 2008) was used to train and test the derived relations.

The contextual cues were arbitrary symbols, whereas the samples and comparisons were either nonarbitrary (i.e., formally related) or arbitrary (i.e., formally unrelated) stimuli, depending on the specific phase. The participants' task was to drag one of five comparisons into a blank comparison square. This was done by placing the cursor over a comparison and holding down the left mouse button. Moving the cursor over the blank square and releasing the left mouse button moved the selected comparison into the "blank" comparison square. The comparison stimulus that was moved was itself simultaneously replaced by a blank yellow square. The sample stimulus remained on-screen throughout the duration of each trial.

When the comparison was dropped, two buttons appeared on the bottom of the screen, with the captions "Finish Trial" and "Start Again" displayed on them. Hovering the cursor over the Finish Trial button produced a small 
text box with the caption "Click here to finish this trial," and hovering over the Start Again button produced the text "Click here to start again." Pressing the Start Again button reset all the stimuli to where they were before the comparison was dropped (i.e., the comparison square on the upper portion of the screen became blank and the selected comparison returned to the lower portion of the screen). Pressing the Finish Trial button cleared the screen and produced the feedback screen during the training phases, and the intertrial interval (ITI) during test phases. During the ITI, which was $3 \mathrm{~s}$ in duration, all stimuli were cleared from the screen and the background color remained blue. The feedback screen background was blue. A yellow box surrounded the sample, the contextual cue, and the selected comparison from the previous trial. If the participant made the correct selection, the word "Correct" was displayed below the yellow box in black type on a yellow background and a beep was presented: otherwise the word "Wrong" was displayed in the same format.

Phase 1: Nonarbitrary relational training. During this phase, all the samples and comparisons were related to each other along a nonarbitrary dimension (e.g., size). Selecting the stimulus that was the farthest away along a particular dimension was reinforced. For example, if the participant was presented with a small cube as the sample and the OPPOSITE contextual cue, then choosing the biggest cube as the comparison was reinforced. The samples and comparisons were all pictures of common objects or shapes. There were six stimulus sets (see Table 1), presented in a purely random order. When participants produced eight consecutively correct responses they were immediately exposed to Phase 2.

Table 1

Stimulus Sets Employed During Phase 1 and Phase 2, and Physical Endpoints of Each Stimulus Set

\begin{tabular}{|c|c|c|}
\hline \multirow[b]{2}{*}{ Description } & \multicolumn{2}{|c|}{ Physical Dimension } \\
\hline & End 1 & End 2 \\
\hline \multicolumn{3}{|l|}{ Phase 1} \\
\hline Red disk sections & Thin crescent & Full disk \\
\hline Lines & Short & Long \\
\hline Cubes & Small & Big \\
\hline Smiley faces & Very sad & Very happy \\
\hline Dots & Few & Many \\
\hline Trees & Small & Big \\
\hline \multicolumn{3}{|l|}{ Phase 2} \\
\hline Buildings & Small & Big \\
\hline Wavy Lines & Small amplitude & Big amplitude \\
\hline Columns & Narrow & Wide \\
\hline Snowstorm & No snow & White-out \\
\hline Bowed trees & Straight & Very bowed \\
\hline Pointed star & Three points & Twenty points \\
\hline
\end{tabular}

Phase 2: Nonarbitrary relational testing. This phase followed the same format as Phase 1, with the exception that no feedback was presented (responses were simply followed by the ITI) and six novel stimulus sets were employed (see Table 1). Participants were required to respond correctly across 
all eight trials to proceed to Phase 3: failure to do so resulted in immediate reexposure to Phase 1.

Phase 3: Arbitrary relational training. During this phase the samples and comparison stimuli were related to each other along an arbitrary dimension (trigrams). The probes for arbitrary relational training and testing are described by using the following convention: the contextual cue is described first in capitals, followed by the sample stimulus, followed by the five comparison stimuli in brackets. The experimenter-designated correct comparison is in italics. For example, the notation SAmE/A1 [B1-B2-N1-N2-N3] indicates that in the presence of the contextual cue SAME and the sample stimulus A1, selecting B1 was reinforced, whereas selecting N1, N2, or N3 was not. All participants were presented with the following four training trials: SAmE/A1 [B1-B2-N1-N2-N3], SAmE/A1 [C1-C2-N1-N2-N3], Opposite/A1 [B1B2-N1-N2-N3], Opposite/A1 [C1-C2-N1-N2-N3]. Training occurred in blocks of eight trials, with each trial type presented twice per block. Participants were required to choose the correct comparison across eight consecutive trials before being exposed to Phase 4 .

Phase 4: Arbitrary relational testing. The aim of this phase was to determine whether responding in accordance with the derived relations of Sameness and Opposition would emerge. Figure 1 shows the predicted relational network. Test trials were not reinforced and were as follows: SAme/B1 [C1-C2-N1-N2-N3], SAme/C1 [B1-B2-N1-N2-N3], SAme/B2 [C1-C2-N1N2-N3], SAme/C2 [B1-B2-N1-N2-N3], Opposite/B1 [C1-C2-N1-N2-N3], Opposite/ C2 [B1-B2-N1-N2-N3], Opposite/B2 [C1-C2-N1-N2-N3], and Opposite/C1 [B1B2-N1-N2-N3]. It is important to note that the present study presented all possible probe trials (i.e., B-C and C-B) during the arbitrary relational test, whereas previous transformation of function studies (e.g., Dymond \& Barnes, 1996; Whelan \& Barnes-Holmes, 2004) have presented the B-C probes only.

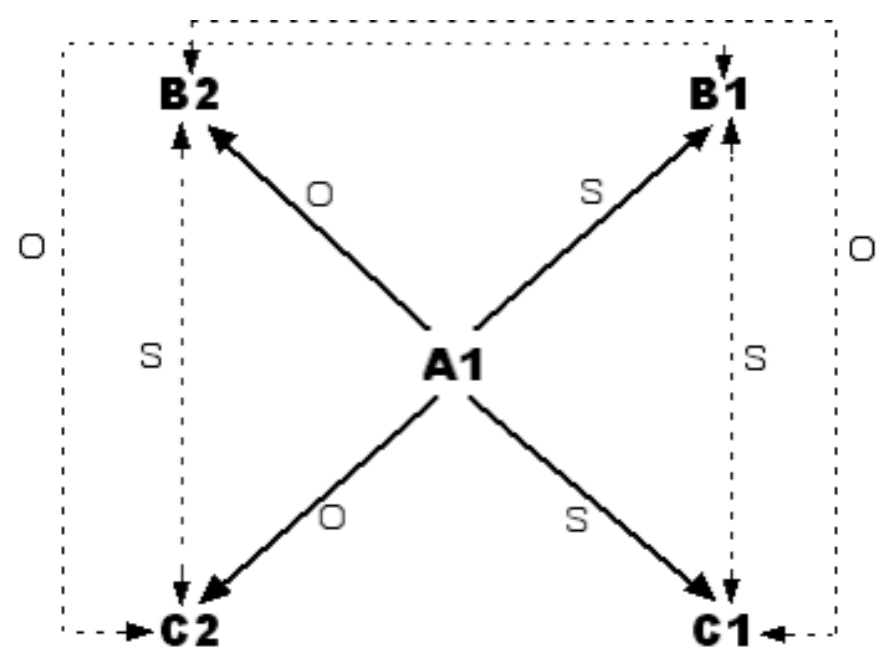

Figure 1. The network of trained and tested stimulus relations. Alphanumerics represent the nonsense syllables used in training and the letters $S$ and $O$ indicate Same and Opposite, respectively. Solid lines indicate trained relations. Dashed lines represent derived relations. 
Responding in accordance with the predicted relational network required that subjects would (a) choose $\mathrm{C} 1$ given $\mathrm{B} 1$ in the presence of SAmE; (b) choose $\mathrm{B} 1$ given $\mathrm{C} 1$ in the presence of SAME ( $\mathrm{C} 1$ and $\mathrm{B} 1$ are both the same as $\mathrm{A} 1$ and therefore the same as each other); (c) choose C2 given B2 in the presence of SAME; (d) choose B2 given C2 in the presence of SAME (C2 and B2 are both opposite to $\mathrm{A} 1$ and therefore the same as each other); (e) choose $\mathrm{C} 2$ given $\mathrm{B} 1$ in the presence of Opposite; (f) choose B1 given C2 in the presence of Opposite (C2 is opposite to $\mathrm{A} 1$, and $\mathrm{B} 1$ is the same as $\mathrm{A} 1$, and therefore $\mathrm{C} 2$ is opposite of B1); ( $\mathrm{g}$ ) choose $\mathrm{C} 1$ given $\mathrm{B} 2$ in the presence of OppositE; and choose $\mathrm{B} 2$ given $\mathrm{C} 1$ in the presence of Opposite $(\mathrm{C} 1$ is the same as $\mathrm{A} 1$, and $\mathrm{B} 2$ is opposite to $\mathrm{A} 1$, and therefore $\mathrm{C} 1$ is opposite to $\mathrm{B} 2$ ).

Testing occurred in a block of sixteen trials, with each task presented twice per block. No feedback was presented after any trial. Participants were required to produce a minimum of $14 / 16$ (i.e., $87.5 \%$ ) correct responses to pass the arbitrary relational test. If this criterion was not met, they were immediately exposed to this training and testing sequence for a predetermined maximum of four exposures.

Phase 5: Avoidance conditioning. The purpose of this phase was to train a simple avoidance response (pressing the space bar) during the presentation of one stimulus (B1) and not during presentations of another stimulus (B2). At the beginning of this phase, participants were given headphones to wear, and the following instructions (modified from Dymond et al., 2007) were presented on the screen:

In a moment, you will be presented with some nonsense words, pictures, and sounds. The pictures and sounds are from real life events and may be considered upsetting to some people. Pictures will be presented on the computer screen and sounds will be presented via headphones.

It is important that you pay attention and concentrate on the screen at all times.

If you have any questions, please ask the experimenter now.

Press any key to continue...

Participants began the first avoidance conditioning trial with a key press. Following the ITI, which varied randomly between $10 \mathrm{~s}$ and $30 \mathrm{~s}$, either the B1 or B2 stimulus appeared in the center of the computer screen for $5 \mathrm{~s}$. A randomly determined ITI was employed to ensure that participants attended to the screen at all times. If participants pressed the space bar while B1 or B2 was present, then the screen cleared and the words "Picture Cancelled" appeared for 2 s. If participants did not press the space bar, the B1 or B2 stimulus was followed by a $2 \mathrm{~s}$ interval after which either a $600 \times 800$ pixel photograph and a sound were presented for $2 \mathrm{~s}$ (following B1) or a photograph was presented for $2 \mathrm{~s}$ (following B2).

Aversive images and sounds followed $75 \%$ of the presentations of B1 when the space bar was not pressed (i.e., $75 \%$ contingency between $B 1$ and the aversive stimuli). A 75\% contingency between B1 and aversive stimuli was employed to prepare participants for the crucial transformation of functions test phase in which the predicted derived $S^{\mathrm{D}}$ for avoidance $(\mathrm{C} 1)$ was never followed by 
aversive stimuli. Previous work showed this contingency to be effective in maintaining avoidance responding. Aversive images and sounds were not presented after the remaining 25\% of presentations of B1 when the space bar was not pressed. Nonaversive images followed $75 \%$ of the presentations of B2 when the space bar was not pressed (i.e., 75\% contingency between B2 and the relief stimulus). Nonaversive images were not presented after the remaining $25 \%$ of presentations of B2 when the space bar was not pressed.

The B1 and B2 stimuli were presented in a quasi-random order (i.e., no more than two consecutive exposures to either) until participants had viewed (i.e., not avoided) a minimum of 20 images. Conditioned avoidance was defined as the production of an avoidance response during each of the final 10 consecutive exposures to $\mathrm{B} 1$ and the absence of an avoidance response during all of the final 10 consecutive exposures to B2. If a participant failed to demonstrate conditioned avoidance according to these criteria, then the tasks were presented once more. This procedure was adopted to ensure that a baseline of avoidance behavior was established prior to the critical transformation test.

Phase 6: Probes for derived avoidance. Phase 6 began immediately after Phase 5. Participants were given a block of 12 trials involving $\mathrm{C} 1$ and $\mathrm{C} 2$ presented in a quasi-random order (with no more than two consecutive presentations of either) until each stimulus had been presented 6 times. Stimuli remained on the screen for 5 s. If a participant pressed the space bar during the presentation of either $\mathrm{C} 1$ or $\mathrm{C} 2$, then the screen cleared and the words "Picture Cancelled" appeared on the screen for 2 s. Failure to press the space bar during the presentation of $\mathrm{Cl}$ was never followed by an aversive image or sound (i.e., 0\% contingency between $\mathrm{C} 1$ and the aversive stimuli), whereas failure to press the space bar during the presentation of $\mathrm{C} 2$ resulted in a 2 s presentation of the relief stimulus (i.e., a $75 \%$ contingency between $\mathrm{C} 2$ and the relief stimulus). Thus, nonavoidance responses to $\mathrm{C} 2$ were followed (75\% of the time) by nonaversive stimuli, but $\mathrm{C} 1$ was presented in extinction.

The predetermined mastery criterion of canceling upcoming images (i.e., emitting the avoidance response) during at least four of the six presentations of $\mathrm{C} 1$ and no more than two of the six presentations of $\mathrm{C} 2$ was used for the derived transformation of avoidance response functions to be said to have occurred. After the 12th trial, a screen appeared with the caption "This is the end of the experiment, please contact the experimenter now."

\section{Results and Discussion}

Table 2 shows the performance of participants during Phases 1 to 4. P5, P8, P9, and P13 passed both the nonarbitrary and arbitrary relational tests on their first exposure. P1, P6, and P11 passed both the nonarbitrary and arbitrary relational tests on their second exposure. $\mathrm{P} 2$ and $\mathrm{P} 4$ required three exposures, and P7 and P12 four exposures to the nonarbitrary and arbitrary relational tests before they met criteria. P12, who initially took 282 trials to meet the nonarbitrary relational training criteria, required multiple training and testing exposures to all phases before passing her fourth and final exposure to the arbitrary relational test. Two participants (P3 and P10) failed to achieve criteria on their maximum fourth exposure to the arbitrary relational test.

All but two of the participants (both of whom had failed the arbitrary 
relational test) progressed to the avoidance-conditioning phase. Participants required between 42 and 102 trials to meet the criteria for conditioned avoidance, while P4 and P8 failed to reach the criteria (Table 3).

\section{Table 2}

Trials to Criterion and Percentage of Correct Responses in Phases 1-4 of Experiment 1

\begin{tabular}{|c|c|c|c|c|}
\hline $\begin{array}{l}\text { Partici- } \\
\text { pant }\end{array}$ & $\begin{array}{l}\text { Phase 1: } \\
\text { Nonarbitrary } \\
\text { Relational Pre- } \\
\text { training (trials } \\
\text { to criterion) }\end{array}$ & $\begin{array}{l}\text { Phase 2: } \\
\text { Nonarbitrary } \\
\text { Relational } \\
\text { Testing (\%) }\end{array}$ & $\begin{array}{l}\text { Phase 3: Arbi- } \\
\text { trary Relational } \\
\text { Training } \\
\text { (trials to } \\
\text { criterion) }\end{array}$ & $\begin{array}{l}\text { Phase 4: Arbi- } \\
\text { trary Relational } \\
\text { Testing (\%) }\end{array}$ \\
\hline \multirow[t]{3}{*}{ P1 } & 11 & 87.5 & & \\
\hline & 8 & 100 & 18 & 50 \\
\hline & 8 & 100 & 13 & 93.75 \\
\hline \multirow[t]{3}{*}{ P2 } & 21 & 100 & 45 & 50 \\
\hline & 8 & 100 & 8 & 62.5 \\
\hline & 8 & 100 & 8 & 100 \\
\hline \multirow[t]{4}{*}{ P3* } & 28 & 100 & 46 & 37.5 \\
\hline & 8 & 100 & 8 & 37.5 \\
\hline & 9 & 100 & 8 & 43.75 \\
\hline & 8 & 100 & 8 & 37.5 \\
\hline \multirow[t]{4}{*}{$\mathrm{P} 4$} & 16 & 100 & 33 & 31.25 \\
\hline & 8 & 75 & & \\
\hline & 8 & 100 & 38 & 43.75 \\
\hline & 8 & 100 & 8 & 87.5 \\
\hline P5 & 13 & 100 & 23 & 93.75 \\
\hline \multirow[t]{2}{*}{ P6 } & 15 & 100 & 54 & 81.25 \\
\hline & 8 & 100 & 9 & 93.75 \\
\hline \multirow[t]{4}{*}{ P7 } & 21 & 100 & 45 & 37.5 \\
\hline & 8 & 100 & 8 & 75 \\
\hline & 8 & 100 & 8 & 81.25 \\
\hline & 8 & 100 & 8 & 87.5 \\
\hline P8 & 8 & 100 & 20 & 87.5 \\
\hline P9 & 14 & 100 & 37 & 87.5 \\
\hline \multirow{4}{*}{ P10* } & 24 & 100 & 25 & 65.25 \\
\hline & 8 & 100 & 8 & 37.25 \\
\hline & 8 & 100 & 8 & 50 \\
\hline & 8 & 100 & 12 & 31.25 \\
\hline \multirow[t]{2}{*}{ P11 } & 14 & 100 & 19 & 81.25 \\
\hline & 8 & 100 & 8 & 93.75 \\
\hline \multirow[t]{6}{*}{ P12 } & 282 & 100 & 48 & 68.75 \\
\hline & 8 & 100 & 8 & 50 \\
\hline & 12 & 100 & 8 & 81.25 \\
\hline & 8 & 87.5 & & \\
\hline & 8 & 87.5 & & \\
\hline & 8 & 100 & 13 & 93.75 \\
\hline P13 & 47 & 100 & 16 & 93.75 \\
\hline
\end{tabular}

*Participants who did not achieve criterion on the predetermined fourth exposure to the arbitrary relational test (Phase 4). 
Of the nine participants who met the criteria for avoidance conditioning and were subsequently tested for derived transformation (Phase 6), three participants (P11, P12, and P13) produced the derived avoidance response during all presentations of $\mathrm{Cl}$ (see Figure 2). P1 and P7 made the derived avoidance response on all but one $\mathrm{C} 1$ trial, and never on $\mathrm{C} 2$ trials. P9 emitted
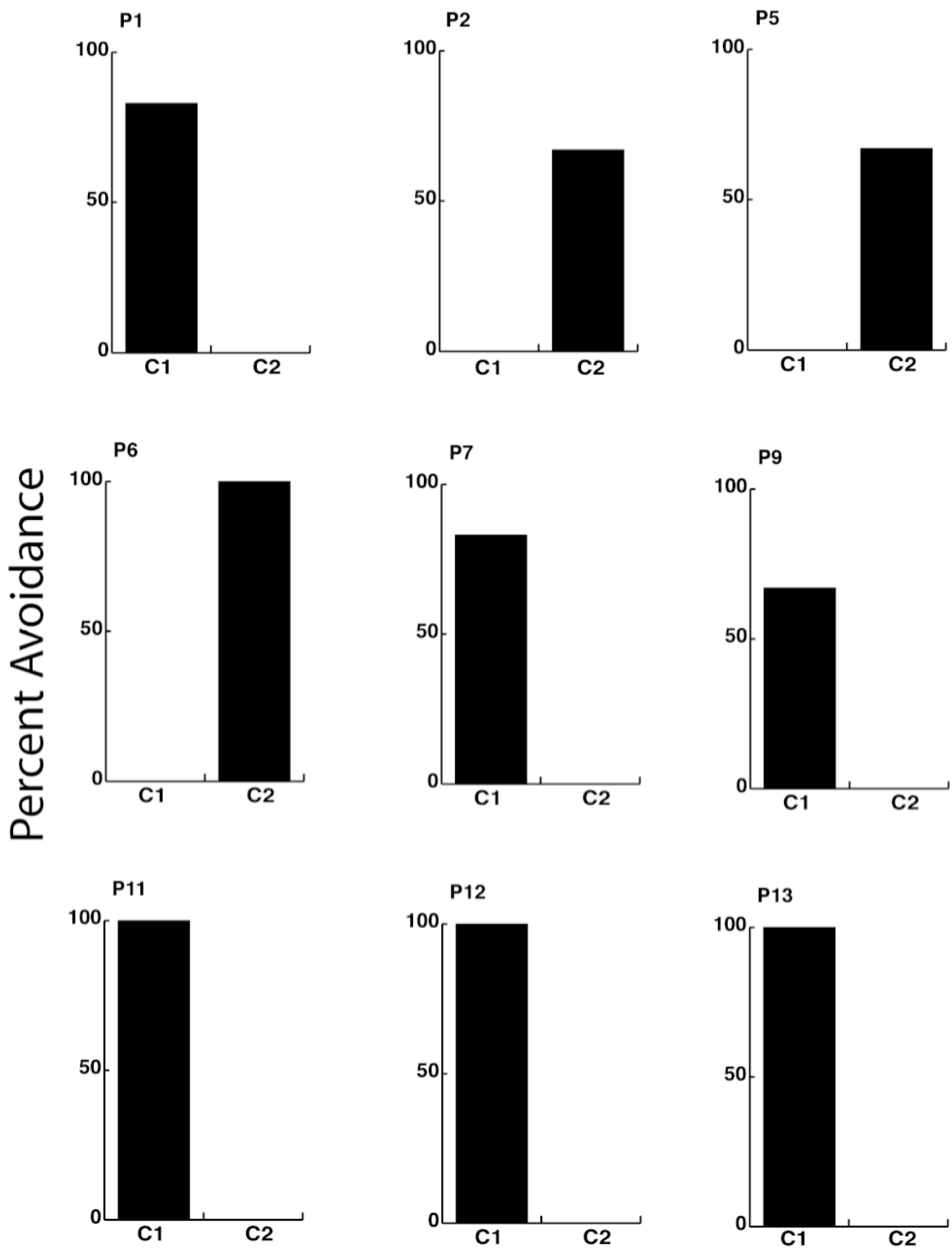

Figure 2. The percentage of trials with an avoidance response to $\mathrm{C} 1$ and $\mathrm{C} 2$, respectively, during the derived transformation test (Phase 6) for those participants who met the criteria for conditioned avoidance in Experiment 1. 
the derived avoidance response on all but two $\mathrm{C} 1$ trials, and never on $\mathrm{C} 2$ trials. Three participants (P2, P5, \& P6) emitted the avoidance response during $67 \%, 67 \%$, and $100 \%$ of $\mathrm{C} 2$ presentations, respectively, and never during C1 presentations.

Overall, six participants met the criteria for derived transformation of avoidance response functions in accordance with Same and Opposite relational frames. The results of Experiment 1 support those of previous studies (e.g., Dymond \& Barnes, 1994, 1998) by demonstrating that detailed instructions are not necessary for derived transformation of avoidance response functions to occur.

Table 3

Avoidance and Nonavoidance Responses During B1 and B2 Presentations and Trials During Avoidance-Conditioning Phase in Experiments 1 and 2

\begin{tabular}{|c|c|c|c|c|c|}
\hline \multirow[b]{2}{*}{ Participant } & \multicolumn{2}{|c|}{ Avoidance Response } & \multicolumn{2}{|c|}{ Nonavoidance Response } & \multirow{2}{*}{$\begin{array}{l}\text { Total No. } \\
\text { of Trials }\end{array}$} \\
\hline & B1 & B2 & B1 & B2 & \\
\hline \multicolumn{6}{|c|}{ Experiment 1} \\
\hline $\mathrm{P} 1$ & 43 & 3 & 8 & 48 & 102 \\
\hline $\mathrm{P} 2$ & 24 & 1 & 1 & 24 & 50 \\
\hline$P 4 *$ & 0 & 0 & 26 & 32 & 58 \\
\hline P5 & 20 & 1 & 2 & 21 & 44 \\
\hline P6 & 12 & 0 & 9 & 21 & 42 \\
\hline P7 & 32 & 5 & 11 & 43 & 91 \\
\hline P8* & 2 & 0 & 24 & 32 & 58 \\
\hline P9 & 20 & 2 & 3 & 20 & 45 \\
\hline P11 & 18 & 0 & 3 & 25 & 46 \\
\hline P12 & 21 & 1 & 2 & 25 & 49 \\
\hline P13 & 17 & 0 & 2 & 23 & 42 \\
\hline \multicolumn{6}{|c|}{ Experiment 2} \\
\hline $\mathrm{P} 14$ & 40 & 0 & 6 & 42 & 88 \\
\hline P15 & 15 & 0 & 7 & 23 & 45 \\
\hline $\mathrm{P} 16$ & 21 & 0 & 3 & 27 & 51 \\
\hline
\end{tabular}

* Participants who did not achieve criterion during the avoidance conditioning phase.

\section{Experiment 2}

Both the findings of Dymond et al. (2007) and Experiment 1 clearly show (a) that avoidance responses may indeed emerge for stimuli by virtue of their participation in complex relational networks of Same and Opposite and (b) that explicit instructions are not necessary either to initiate contact with the avoidance schedule or for derived transformation to occur. Next, in Experiment 2, we sought to more fully examine the patterns of stimulus transformation that might occur via the relational network when the $S^{\mathrm{D}}$ for avoidance was varied. Previously, avoidance responses were trained in the presence of B1 and transformation tested by presentations of C1. Successful performance on the derived transformation test involves a combinatorially entailed relation of sameness between $\mathrm{B} 1$ and $\mathrm{C} 1$. (B1 and $\mathrm{C} 1$ were both trained as being the same as Al and therefore the same as each other; see Figure 1.) In Experiment 2, avoidance responses were trained in the presence of B2 and transformation tested with C2 (both B2 and C2 are the opposite to 
A1 and therefore the same as each other; see Figure 1). In effect, the current experiment should generate derived avoidance in accordance with two Opposite relations (see also Roche and Barnes, 1997).

\section{Method}

\section{Participants}

Three participants, aged between 21 and 38 years of age, were recruited via bulletin board announcements or personal contacts from the University of Wales, Swansea, and were paid $£ 5$ after completion of the study.

\section{Procedure}

The procedure for Experiment 2 was identical to that for Experiment 1 except for the following important difference. In Phase 5 (avoidance conditioning), B2, and not B1, signaled the avoidance response. If participants pressed the space bar while either stimulus was present, then the screen cleared and the words "Picture Cancelled" appeared. If participants did not press the space bar, the B1 or B2 stimulus was followed by a photograph or sound, or both. Aversive images and sounds followed 75\% of the presentations of B2 when the space bar was not pressed. Nonaversive images followed $75 \%$ of the presentations of B1 when the space bar was not pressed.

\section{Results and Discussion}

Table 4 shows the performance of participants during Phases 1 to 4 . P14 and P15 passed both the nonarbitrary and arbitrary relational tests on their first exposure. P16 required three exposures to the nonarbitrary relational test and two exposures to the arbitrary relational test before meeting the criteria.

Table 4

Trials to Criterion and Correct Responses in Phases 1-4 in Experiment 2

\begin{tabular}{|c|c|c|c|c|}
\hline Participant & $\begin{array}{l}\text { Phase 1: } \\
\text { Nonarbitrary } \\
\text { Relational } \\
\text { Pretraining } \\
\text { (trials to } \\
\text { criterion) }\end{array}$ & $\begin{array}{l}\text { Phase 2: } \\
\text { Nonarbitrary } \\
\text { Relational } \\
\text { Testing (\%) }\end{array}$ & $\begin{array}{l}\text { Phase 3: } \\
\text { Arbitrary } \\
\text { Relational } \\
\text { Training } \\
\text { (trials to } \\
\text { criterion) }\end{array}$ & $\begin{array}{l}\text { Phase 4: } \\
\text { Arbitrary } \\
\text { Relational } \\
\text { Testing (\%) }\end{array}$ \\
\hline P14 & 22 & 100 & 35 & 100 \\
\hline P15 & 19 & 100 & 18 & 87.5 \\
\hline \multirow[t]{4}{*}{ P16 } & 119 & 62.5 & & \\
\hline & 8 & 75 & & \\
\hline & 8 & 100 & 38 & 43.5 \\
\hline & 8 & 100 & 8 & 87.5 \\
\hline
\end{tabular}

Table 3 shows that all participants in Experiment 2 required between 45 and 88 trials to meet the criteria for conditioned avoidance. During the test 
for transformation, P16 emitted the derived avoidance response on all but one C2 trial and never during C1 trials. Both P14 and P15 emitted the derived avoidance response on all but two C2 trials and, for P15 only, on one C1 trial (see Figure 3).
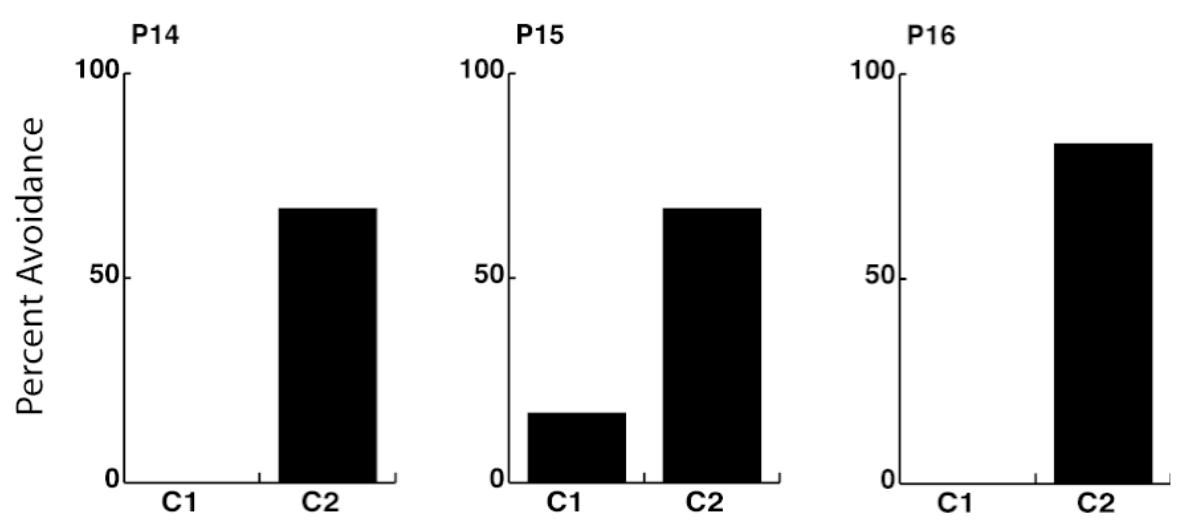

Figure 3. The percentage of trials with an avoidance response to $\mathrm{C} 1$ and $\mathrm{C} 2$, respectively, during the derived transformation test (Phase 6) for those participants who met the criteria for conditioned avoidance in Experiment 2.

\section{General Discussion}

The findings of Experiment 1 showed that detailed instructions were not necessary either to initiate contact with the avoidance contingency or for derived transformation to occur. The findings of Experiment 2 showed that complex forms of transformation may emerge when the stimulus used from the relational network to signal the aversive events is varied. Overall, the findings of the two experiments replicate and extend the findings of Dymond et al. (2007).

During the derived transformation test, nonaversive stimuli were presented after $75 \%$ of C2 trials, and thus it may be argued that participants did not produce derived nonavoidance responses during the probe phase. This procedure was employed in an attempt to change as little as possible the task format from avoidance conditioning to testing for derived avoidance. Had C2, like C1, also been presented in extinction (i.e., not followed by any stimuli, aversive or otherwise; Delamater, 2004), the sudden change in task format may have functioned as a contextual cue for "the experiment is over" and participants' responses may have been severely disrupted.

Given the foregoing procedure it might be further suggested that participants also failed to produce genuine derived avoidance responses to C1. More specifically, given a history of emitting avoidance responses during one stimulus and not during the other in the conditioning phase, participants may have quickly discovered that presentations of C2 were, $75 \%$ of the time, followed by nonaversive stimuli and therefore concluded that presentations of $\mathrm{C} 1$ would be followed by aversive stimuli unless an avoidance response was emitted. This suggestion might indeed be plausible if acquisition was observed in the avoidance responses to $\mathrm{Cl}$ and the nonavoidance responses to C2. However, this clearly was not the case for the following reasons. First, 
the majority of participants produced the predicted avoidance response to $\mathrm{C} 1$ (or C2 in Experiment 2) from the very first probe trial. Second, the majority of participants produced the nonavoidance response to every presentation of C2. Finally, because $\mathrm{C} 1$ was presented in extinction the avoidance responses never had opportunity to come under direct contingency control. Thus, participants were not simply responding to a direct, rather than a derived, contingency during the probe trials.

The use of a negative reinforcement schedule during avoidance conditioning and the 75\% contingency between stimuli and aversive events seems to have rendered the response patterns resistant to extinction. This finding is particularly interesting given that a robust pattern of derived avoidance was observed across a total of twelve probe trials. Indeed, future studies might further examine this robustness by testing for derived avoidance across a greater number of probe trials. The long-term stability or maintenance of derived transformation also warrants further attention (see Rehfeldt \& Dymond, 2005).

As outlined earlier, the present findings may have implications for understanding indirectly acquired fear and avoidance. First, the ease with which participants formed the derived relations of Same and Opposite using the relational completion procedure, and the persistence of the avoidance responses during the critical probes phase, highlights the difficulty of breaking apart such relational responses once established. In this way, the stimulus functions attached to, or fused with, members of a relational network may become highly resistant to extinction and in practical terms rarely go away but instead become further elaborated. This account suggests one reason why persons with successfully treated anxiety disorders may, in turn, relapse after therapy. It also suggests that therapists using exposure-based interventions may need to alter the contexts holding such relations intact, perhaps by teaching clients to discriminate their relational responding without acting on the functions of such relations (see Eifert \& Forsyth, 2005, for a relevant example of a behavior-analytic protocol for anxiety disorders).

Second, it is likely that exposure to the trained aversive stimulus may attenuate the aversive functions of other stimuli in the relational network. However, this attenuation may be temporary when novel contexts occasion other members of the relational network and result in the "return of fear" seen clinically (e.g., Bouton, Mineka, \& Barlow, 2001). Thus, in a therapeutic context it would seem important to arrange exposures to capitalize on contextual variation and its effects on relational responding. This might be achieved by targeting directly the tendency toward experiential avoidance, teaching clients new ways of relating to their own private events, or helping clients to be less rule-governed and more sensitive to prevailing contingencies as they are and not what their history tells them that they are (Eifert \& Forsyth, 2005; Hayes, 2004).

Finally, the present approach offers a novel means of examining the complex verbal, relational aspects of many clients' avoidance repertoires and suggests a functional-analytic interpretation of clients' rationales for avoidance in terms of the transformation of stimulus functions. Indeed, opposition relations seem critical in the derived establishment of safety and avoidance behaviors across the anxiety disorders. More specifically, anxious clients readily learn what does or may evoke fear and anxiety, and seem to quickly identify situations, contexts, and behaviors that are 
safe (i.e., opposition relations). Consequently, they spend considerable time engaging in safe behaviors that are the opposite of (i.e., exclusive of) potentially fear evoking but often worthwhile and desirable activities. Such issues have been relatively understudied to date and appear to warrant further investigation.

Future research should address potential limitations of the current findings such as the variability in individual performance, during both the relational training and testing phases and the avoidance conditioning and transformation testing phases, and the low number of participants in Experiment 2 relative to Experiment 1 (and that of Dymond et al., 2007). A pre-experimental assessment of the perceived aversive functions of the IAPS and IADS stimuli should also be considered.

In conclusion, the present procedures and findings would appear to offer a powerful and technically sophisticated approach to the analysis of anxiety and avoidance that supplements basic research on avoidance conditioning and derived relations. Of course, the current study represents just one example of how a relational frame-based approach to the empirical analysis of derived fear and avoidance might proceed, but in so doing it has opened an avenue of research that is well worth pursuing.

\section{References}

AUGUSTSON, E. M., \& DOUGHER, M. J. (1997). The transfer of avoidance evoking functions through stimulus equivalence classes. Journal of Behavior Therapy and Experimental Psychiatry, 3, 181-191.

BOUTON, M. E., MINEKA, S., \& BARLOW, D. H. (2001). A modern learning theory perspective on the etiology of panic disorder. Psychological Review, 108, 4-32.

BRADLEY, M. M., \& LANG, P. J. (1999). International affective digitized sounds (IADS): Stimuli, instruction manual, and affective ratings (Tech. Rep. No. B-2). Gainesville: Center for Research in Psychophysiology, University of Florida.

DAWSON, M. E., \& SCHELL, A. M. (1987). Human autonomic and skeletal classical conditioning: The role of conscious cognitive factors. In G. Davey (Ed.), Cognitive processes in Pavlovian conditioning in humans (pp. 27-55). London: Wiley.

DEHOUWER, J., VANDORPE, S., \& BECKERS, T. (2004). On the role of controlled cognitive processes in human associative learning. In A. J. Wills (Ed.), New directions in human associative learning (pp. 41-63). Mahwah, N.J.: Lawrence Erlbaum.

DELAMATER, A. R. (2004). Experimental extinction in Pavlovian conditioning: Behavioral and neuroscience perspectives. Quarterly Journal of Experimental Psychology, 57B, 97-132.

DYMOND, S., \& BARNES, D. (1994). A transfer of self-discrimination response functions through equivalence relations. Journal of the Experimental Analysis of Behavior, 62, 251-267.

DYMOND, S., \& BARNES, D. (1996). A transformation of self-discrimination response functions in accordance with the arbitrarily applicable relations of sameness and opposition. Psychological Record, 46, 271-300.

DYMOND, S., \& BARNES, D. (1997). Behavior analytic approaches to selfawareness. Psychological Record, 47, 181-200.

DYMOND, S., \& BARNES, D. (1998). The effects of prior equivalence testing 
and detailed verbal instructions on derived self-discrimination transfer: A follow-up study. Psychological Record, 48, 147-170.

DYMOND, S., \& REHFELDT, R. (2000). Understanding complex behavior: The transformation of stimulus functions. Behavior Analyst, 23, 239-254.

DYMOND, S., ROCHE, B., FORSYTH, J. P., WHELAN, R., \& RHODEN, J. (2007) Transformation of avoidance response functions in accordance with the relational frames of Same and Opposite. Journal of the Experimental Analysis of Behavior, 88, 249-262.

DYMOND, S., \& WHELAN, R. (2008). Derived relational responding: A comparison of matching-to-sample and the relational completion procedure. Manuscript submitted for publication.

EIFERT, G. H., \& FORSYTH, J. P. (2005). Acceptance \& commitment therapy for anxiety disorders: A practitioner's treatment guide to using mindfulness, acceptance, and values-based behavior change strategies. Oakland, CA: New Harbinger.

FIELD, A. P. (2006). Is conditioning a useful framework for understanding the development and treatment of phobias? Clinical Psychology Review, $26,857-875$.

FORSYTH, J. P., EIFERT, G. H., \& BARRIOS, V. (2006). Fear conditioning research as a clinical analog: What makes fear learning disordered? In M. G. Craske, D. Hermans, \& D. Vansteenwegen (Eds.). Fear and learning: Basic science to clinical application (pp. 133-153). Washington, DC: American Psychological Association.

HAYES, S. C. (2004). Acceptance and commitment therapy, relational frame theory, and the third wave of behavioral and cognitive therapies. Behavior Therapy, 35, 639-665.

LANG, P. J., BRADLEY, M. M., \& CUTHBERT, B. N. (2005). International affective picture system (IAPS): Affective ratings of pictures and instruction manual (Tech. Rep. No. A-6). Gainesville: University of Florida.

LEJUEZ, C. W., O'DONNELL, J., WIRTH, O., ZVOLENSKY, M. J., \& EIFERT, G. H. (1998). Avoidance of $20 \%$ carbon dioxide-enriched air with humans. Journal of the Experimental Analysis of Behavior, 70, 79-86.

LOVIBOND, P. (2006). Fear and avoidance: An integrated expectancy model. In M. G. Craske, D. Hermans, \& D. Vansteenwegen (Eds.). Fear and learning: Basic science to clinical application (pp. 117-132). Washington, DC: American Psychological Association.

LOVIBOND, P., \& SHANKS, D. (2002). The role of awareness in Pavlovian conditioning: Empirical evidence and theoretical implications. Journal of Experimental Psychology: Animal Behavior Processes, 28, 1-26.

RACHMAN, S. J. (1977). The conditioning theory of fear acquisition: A critical examination. Behaviour Research and Therapy, 15, 375-387.

RACHMAN, S. J. (1991). Neo-conditioning and the classical theory of fear acquisition. Clinical Psychology Review, 11, 155-173.

REHFELDT, R., \& DYMOND, S. (2005). The effects of test order and nodal distance on the emergence and maintenance of derived discriminative stimulus functions. Psychological Record, 55, 179-196.

ROCHE, B., \& BARNES, D. (1997). A transformation of respondently conditioned function in accordance with arbitrarily applicable relations. Journal of the Experimental Analysis of Behavior, 67, 275-301. 
STEELE, D., \& HAYES, S. C. (1991). Stimulus equivalence and arbitrarily applicable relational responding. Journal of the Experimental Analysis of Behavior, 56, 519-555.

WHELAN, R., \& BARNES-HOLMES, D. (2004). The transformation of consequential functions in accordance with the relational frames of same and opposite. Journal of the Experimental Analysis of Behavior, 82, 177-195.

WILSON, K. G., \& BLACKLEDGE, J. T. (2000). Recent developments in the behavioral analysis of language: Making sense of clinical phenomena. In M. J. Dougher (Ed.), Clinical behavior analysis (pp. 27-46). Reno, NV: Context Press. 